\title{
The Term Prairie in the United States
}

\author{
E. Wallace McMullen
}

True Generics

$\prod^{1}$

HE PURPOSE OF THIS ARTICLE is to discuss the application of the term prairie in the United States on the basis of current evidence of geographical distribution. ${ }^{1}$ The information presented here is the result of a preliminary study of names containing the word prairie on 773 topographical maps published by the U. S. Geological Survey, the U. S. Army Map Service, the Mississippi River Commission, and the TVA, ranging in date from 1901 to 1955 and including some unpublished maps still in the proof stage. These 773 maps, which constitute all the maps of areas in the United States on which prairie is recorded, were made available to me by Dr. Meredith F. Burrill, to whom I am indebted for having suggested the project, for placing his own files at my disposal, and also for giving time and counsel to furthering this undertaking. I have consulted him on matters of technical interpretation, and, with his permission, am reproducing his map which shows the geographical distribution of prairie throughout the country.

The term prairie is used on topographical maps in all but fifteen states which include all the New England and Atlantic states (except New York) down to Georgia, and also West Virginia, Utah and Nevada. (Here I am referring to both true and false generics.) Thus, at present we have no map evidence that prairie generics are used in the states just referred to. ${ }^{2}$ One reason is that the eastern

1 With minor alterations read as a paper for the American Names Society meeting during the 1955 MLA convention in Chicago.

2 On this subject the writer is checking at present with the Linguistic Atlas authorities; and he would welcome any information on prairie terms from anyone else.

Professor H. F. Raup of Kent State University has kindly furnished me with his list of prairies (both true and false generics) in the state of Ohio which, as he says, occur in earlier material as far back as 1841. None of Professor Raup's terms appeared on the current topographical maps, excepting one, Sunbeam Prairie 
area is forested, with few spots of natural non-arboreal vegetation except the wetlands to which other terms are applied. However, probably everybody in America knows the word prairie itself.

According to Dr. Burrill prairie has much the same pattern of occurrence on the topographical maps as other French topographic expressions like coulee. Evidently prairie was carried into the Mississippi Valley by early French pioneers and on out the northwest and down the Pacific coast as far as northern California. But prairie seems to differ from coulee in extending east of the Mississippi Valley and down along the Gulf coast and into southern Florida.

There are 367 named prairies on the source maps, and 95 of these occur in Washington, more than in any other state. There are 66 in Oregon, 52 in California, one each in Indiana, New Mexico, and Ohio, and no true generics at all in twenty-one states. Incidentally, Illinois - which is called the Prairie State - has only nine. Perhaps this paucity of occurrence where we would expect the opposite is due to the human tendency not to specify the obvious. Dr. Burrill tells me there are comparatively few map examples of the term valley as a true generic in the Mississippi Valley area, probably, he believes, for the reason just given. Another explanation for the lack of named prairies in the Illinois region is that after agricultural settlement, prairies lost their original characteristics.

Although several different types of prairies are charted on the topographical maps, the impression one receives from this fact may very well be erroneous. This apparent variety may be explained as either a difference in investigators, or a change in condition of the terrain subsequent to field observation - that is, what may have looked dry to a person on foot, later on, after rainfall, might have photographed from the air as a lake. At any rate, for purposes of classification I divided the states into those whose prairies

(New Paris), and some of the false generics. However, I am indebted to him for his true generics, which follow: Big Spring Prairie, Freemans Prairie, Gahagans Prairie, Gehagan Prairie, Gerard Prairie, Gibsons Prairie, Hulls Prairie, Hull Prairie, Jennings Prairie, Johnston Prairie, Pickawillany Prairie, Liberty Prairie, Mud Creek Prairie, Pee Pee Prairie, Richie Prairie, Shanes Prairie, Tontogany Prairie, Twenty Seven Mile Prairie, Williams Prairie, and Sunbeam Prairie. Professor Raup mentions Belpre, a township in Ohio, the second element of which stems from the same Latin word as the original of prairie. Strictly speaking, though, a consideration of -pre does not fall within the limits of this article. 
are recorded as dry, those with partly or wholly wet prairies, and those with both wet and dry prairies. Ohio, Illinois, Oklahoma, Mississippi, New Mexico, Arizona, South Dakota, Wyoming, Idaho and California are dry-prairie states. The saturated prairie states are Florida, Georgia, Indiana and North Dakota. Wisconsin, Minnesota, Missouri, Arkansas, Louisiana, Texas, Montana, Oregon,

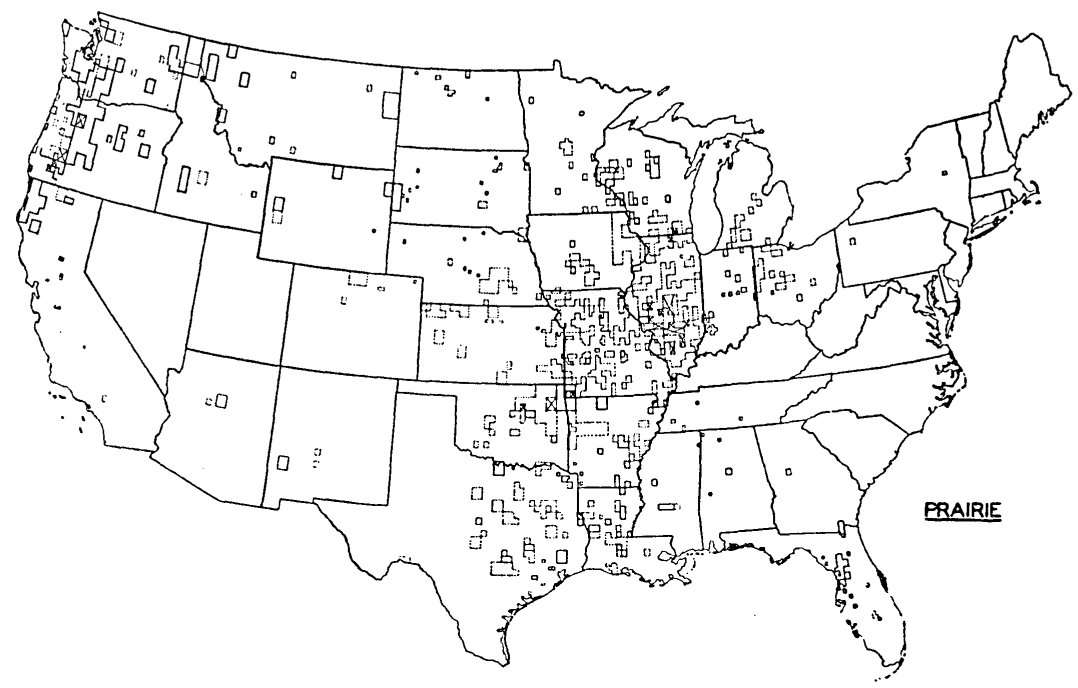

MAP SHOWING THE LOCATION OF TOPOGRAPHICAL MAPS IN THE UNITED STATES ON WHICH PRAIRIE GENERICS WERE FOUND

Solid lines enclose true generics. Dotted lines enclose false generics. Crossed lines signify enclosed areas of nonoccurrence.

(Map drawn by Meredith F. Burrill; reproduced by permission)

and Washington seem to have both wet and dry prairies. But, as I said, these categories should not be too strictly applied, since a prairie is essentially a grassy open area usually moist or subject to occasional overflow.

According to the map evidence, prairies occur in or near water areas and also on poorly drained areas, in Florida; on the bottom lands of the upper Mississippi river, in the Illinois-Iowa region; on flat areas above stream level, from 5 to 20 feet in Louisiana, and somewhat higher in Illinois and the adjacent states; and on the terraces of the Spokane area, where they resemble mountain meadows. 
One particularly puzzling type of prairie recurs on the maps of three states in what look like completely wooded areas. Though dense forests may not cover these spots, the presence of trees there is indicated on sixteen maps in Washington, three in Oregon and one in California.

A good number of prairie names illustrate some of the foreign influences on our culture. In this respect France has contributed the most, the French terms in prairie combinations being illustrated by Belle Prairie, ${ }^{3}$ Prairie des Femmes, Grande Cote Prairie, Avoyelles Prairie, Prairie Ronde, Boistfort [?] Prairie, Latourelle Prairie, Mashel [?] Prairie, Prairie du Sac, Pepin [?] Prairie, Bayou Petite Prairie, BM Petite Prairie, Prairie de Butte, Prairie Mer Rouge, and Maumelle [?] Prairie.

Almost as many of the names come from American Indian, such as Skookum Prairie, Payne (name of an Indian chief in Florida) Prairie or Alachua Lake, Kanapaha Prairie, Chelatchie Prairie, and Swauk [?] Prairie. Perhaps here should also be included Indian Prairie and Indian Lake Prairie.

Spanish names occur in Canyon Springs Prairie, Peone [?] Prairie, and Sanchez Prairie.

Amsterdam Prairie, Alpha Prairie and Elos (Ancient Greek for "marsh") Prairie are the only examples in this material of Dutch and Greek influence, respectively.

Several prairies appear to be connected with the history of a region, such as Empire, Territory, Centennial, Courthouse, 91 st Division, 13th Division, 4th Division, Old Pearl, Pitt Place, and Pe Ell (which is probably a brand name). Others seem to be linked with some incident or occasion like Christmas, Bark Shanty, Burnt Ranch, Lately, Lost, Lonesome, Bridge, Coffee Frost [ ?], Diamond Grove, Sunbeam, Cowskin, Happy, Bottle, Fingerboard and Tent Prairie.

A great number of prairies are probably named for persons, and make up by far the largest of the categories. This group ${ }^{4}$ includes

Washington, Payne (also known as Alachua Lake), Flannigan, Bailey, Bristow, Caldwell, Kit Price, Joes, Vasher, Garland, Earl,

3 The geographical location of the prairie names is given at the end of the article.

4 This particular list is arranged for the most part according to states. See the geographical listing at the end of the article. 
Lindsley, Norwood, Touchstone, Kings, Boynton, Heustis, Dolason Hill, Thompson, Smith, Ford, Shelton, Hancorn, Barber, Johnson, Jones, Dunn, Gans, Moores, Harrow, Stevens, Eukers, Hancorne, Chadd, Luke, Sanchez, Gooski, Sturgis, Ross, May, Evans, Levys, Putnam, Goodson, Ashley, Priest, Hickman, Floyd Island, Carters, Durdin, Chase, Christie, Mizell, Rathdrum, String, Eden, Barnes, Rollison, Handley, Britton, Olivers, Burkhart, Spurgeon, Brock, Joys, Pools, Lanes, Swars, Tom Lay, Cooney, Yankee Bill, Jackson, Picket, Donegan Prairies (sic), Abbott, Hall, Smock[?], Walker, Camas, Jeeter, Perkins, Jerome, Crane, Gray, Lytle, Whitten, Dayton, Marks, Gribble, Dickey, Howell, Elliott, Gibson, Major, Wilson, Gutherie, Pickett, Reynolds, Garden [ ?], Gilette, Peters, Caughorn, Hemphill, Hills, Crafts, Tarkington, Batson, Cobb, Wheelock, Paynes, Fords, Boistfort, Warner, German [ ?], Latourell [ ?], Lacamas, Drews, Layton, Ebeys, Chambers, Carstairs, Hawks, Little Chambers, Connells, Bachelor, Cash, Ahlstroms, Rooses, Mannys, Baker, Mima, Jolm, Moran, Frost [?], Viblet (sic), Waunch, Ruth, Weir, Dalley, and Yelm Prairies.

Many American prairies are associated with animals, as illustrated by Bear, Beaver Creek, Buck, Bull, Calf Ranch, Cowskin, Dog, Dry Cow House, Eagle, Elk, Fawn, Fish, Fox, Goose, Grasshopper, Hog Ranch, Hogtown, Horse, Jackass, Pigeon, Wildhorse and Wolf Prairies.

Prairies having to do with elevation are called High, Counts Hill, Sidehill, Mound, Grand Mound, Four Mound, Ridge, Cagle Ridge, Summit, Big Summit and Little Summit Prairies.

Those prairies with modifying elements which designate various types of vegetation are Bee Gum, Brush, Brushy, Bush, Crabapple, Fern, Grass, Hawthorn, Indigo, Postoak, Thorn, Wheatland, Wild Rose and Wiregrass Prairies. Terms like Pine Prairie pose a problem, for prairies usually lack trees. Perhaps an open pine forest is located on such an area. Lone Pine Prairie is probably named for a solitary tree on the grounds, and Bald Prairie is probably so called from the lack of vegetation.

Only three of the examples refer to land surrounded by water, namely, Floyd Island Prairie, Pine Island Prairie and Little Pine Island Prairie.

Three prairies are named for streams - Beaver Creek, Forks, and Canyon Springs Prairies. The quality of water is indicated by Sweetwater Prairie. 
Bodies of water are denoted by Payne Prairie which is also known as Alachua Lake, and the qualifying components of Lake, Dead Lake, Keith Lake, Spring Lake, and Pond Prairies. However, according to the maps Fish, Church, Mud and Hickman Prairies either are lakes or appear to be lakes. Gooski Prairie and Black Sink Prairie are also mapped as water with vegetation around the edges.

The shape of terrain is specified in Round, Flat, and Half Moon Prairies; and the character of the soil is denoted by the modifying portions of Sand, Gravel, Cinder, Iodine, Rock, Rocky, White Rock, Saline, Salt Licks, Mud, and Slate Prairies.

General and unusual expressions, or terms which do not easily lend themselves to analysis, include The Prairie, and Long, Big, Little, String, Racetrack, Blossom, Government, Grand, May, New, Bottle, Floating, Line (borderline [?]), North, and Smock Prairies. A few prairies are designated by terms of measurement, like Six Mile, Eightmile, and Nine Mile Prairies.

Probably the most significant and most interesting single characteristic of prairie - and, for that matter, all other topographic terms - stands out after an examination of the examples given above: namely, the great freedom with which it combines with a wide variety of expressions - foreign, historical, natural, and others.

Oddly enough, a connection between false generics and true generics is generally not shown on topographical maps. For example, on the same map where Horse Prairie Creek is recorded there is no sign whatsoever of a Horse Prairie. However, if there were more investigations of this type we would better understand some etymological details and other aspects of the linguistic behaviour of this body of terms than we do at present.

The study of prairie generics not only provides us with a good example of French influence on the current American vocabulary but also indicates the approximate geographical areas where that influence has been the greatest. Yet in that part of the country which we usually think of as the prairie region it is surprising to find very few prairies so called.

The prairies referred to, including identical forms, and the topographical maps on which they occur, are listed below: 
Arizona:

Garland Prairie (Flagstaff)

Government Prairie (Flagstaff)

Arkansas:

Earl Prairie (Booneville)

Long Prairie (England)

Lindsley Prairie (Fayetteville)

Norwood Prairie (Fayetteville)

Coffee Prairie (Fayetteville)

Pine Prairie (Ingalle)

Maumelle Prairie (Leachville)

Grand Prairie (Lonoke)

Touchstone Prairie (Rotan)

Grand Prairie (Stuttgart)

Long Prairie (Stuttgart)

Pine Prairie (Wilmot)

Brushy Prairie (Wilmot)

Kings Prairie (Yellville)

California:

Round Prairie (Blue Lake)

Elk Prairie (Blue Lake)

Fern Prairie (Blue Lake)

Christmas Prairie (Blue Lake)

High Prairie (Blue Lake)

Boynton Prairie (Blue Lake)

Heustis Prairie (Blue Lake)

Lake Prairie (Blue Lake)

Long Prairie (Blue Lake)

Fawn Prairie (Blue Lake)

Long Prairie (Bray)

Pitt Place Prairie (Coyote Peak)

Dolason Hill Prairie (Coyote Peak)

Thompson Prairie (Coyote Peak)

Smith Prairie (Coyote Peak)

Bear Prairie (Coyote Peak)

Sidehill Prairie (Coyote Peak)

Big Prairie (Coyote Peak)

Ford Prairie (Coyote Peak)

Shelton Prairie (Coyote Peak)

Cagle Ridge Prairie (Coyote Peak)
Counts Hill Prairie

(Coyote Peak)

Childs Hill Prairie

(Coyote Peak)

Hancorn Prairie (Coyote Peak)

Skookum (Coyote Peak)

Barber Prairie (Coyote Peak)

Johnson Prairie (Coyote Peak)

Long Prairie (Coyote Peak)

100 Acre Prairie (Coyote Peak)

Hog Ranch Prairie

(Coyote Peak)

Wiregrass Prairie (Coyote Peak)

Burnt Ranch Prairie

(Coyote Peak)

Jones Prairie (Fortuna)

Sandy Prairie (Fortuna)

Lake Prairie (Hoopa)

Fern Prairie (Hoopa)

Dunn Prairie (Hoopa)

Grasshopper Prairie (Hoopa)

Iodine Prairie (Medicine Lake)

High Prairie (Oregon House)

Gans Prairie (Orick)

Rock Prairie (Orleans)

Moores Prairie (Scotia)

Johnson Prairie (Scotia)

Harrow Prairie (Scotia)

Stevens Prairie (Tectah Creek)

Eukers Prairie (Tectah Creek)

Hancorne Prairie (Tectah Creek)

Elk Prairie (Weott)

Chadd Prairie (Weott)

Horse Prairie (Weott)

Luke Prairie (Weott)

Johnson Prairie (Weott)

Johnson Prairie (Willow Creek)

Florida:

Payne Prairie or Alachua Lake (Arredondo)

Kanapaha Prairie (Arredondo)

Grass Prairie (Arredondo) 
Hogtown Prairie (Arredondo)

Sanchez Prairie (Arredondo)

Indian Lake Prairie (Citra)

Gooski Prairie (Citra)

Black Sink Prairie (Citra)

Hawthorn Prairie (Citra)

Sturgis Prairie (Cotton Plant)

Ross Prairie (Dunnnellon SE)

May Prairie (Nobleton)

Spring Lake Prairie (Nobleton)

Eagle Prairie

(Okefenokee Slough)

Evans Prairie (Panasoffkee)

Levys Prairie (Putnam Hall)

Putnam Prairie (Putnam Hall)

Goodson Prairie (Putnam Hall)

Ashley Prairie (Putnam Hall)

Pine Island Prairie (Romeo)

Little Pine Island Prairie (Romeo)

Dry Cow House Prairie (Romeo)

Priest Prairie (Williston)

Fish Prairie (Williston)

Church Prairie (Williston)

Mud Prairie (Williston)

Hickman Prairie (Williston)

Georgia :

Floyd Island Prairie (Folkston)

Carters Prairie (Folkston)

Durdin Prairie (Folkston)

Territory Prairie (Folkston)

Chase Prairie (Folkston)

Christie Prairie (Folkston)

Mizell Prairie (Folkston)

Mizell Prairie (Moniac)

Grand Prairie (Moniac)

Bee Gum Prairie (Moniac)

Idaho:

Kit Price Prairie (Cataldo)

Jackass Prairie (Cataldo)

Jackass Prairie

(Coeur d'Alene District)
Little Prairie (Craters of the Moon National Monument)

Smith Prairie (Idaho City)

Smith Prairie (Mountain Home)

Eightmile Prairie (Rathdrum)

Rathdrum Prairie (Rathdrum)

Illinois:

Grand Cote Prairie (Coulterville)

Flat Prairie (Coulterville)

Lost Prairie (Coulterville)

Six Mile Prairie (Coulterville)

Belle Prairie (McLeansboro)

North Prairie (Nashville)

Ridge Prairie (O'Fallon)

Old Pearl Prairie (Pearl)

String Prairie (Roodhouse)

Indiana:

Fox Prairie (Noblesville)

Kansas:

Belle Prairie (Ness)

Louisiana:

Prairie de F'emmes (Arnaudville)

Avoyelles Prairie (Marksville)

Prairie Ronde (Opelousas)

Bayou Petite Prairie (Odenburg)

The Prairie (Calvin S. W.)

Petite Prairie (Palmetto)

Little Prairie (Buckeye)

Vasher Prairie (Buckeye)

Long Prairie (Buckeye)

Big Prairie (Colfax)

Boeuf Prairie (Harrisonburg)

Racetrack Prairie (Logansport)

Gravel Prairie (Logansport)

Prairie de Butte (Naff)

Prairie Mer Rouge (Naff)

Minnesota:

Eden Prairie (Eden Prairie)

Mississippi :

Barnes Prairie (Jackson)

Bald Prairie (Jackson) 
Belle Prairie (Mileston)

Bald Prairie (Morton)

Line Prairie (Morton)

Barnes Prairie (Pelahatchie)

Round Prairie (Pelahatchie)

Rollison Prairie (Pelahatchie)

The Prairie (Grand Bay)

Missouri:

Handley Prairie (Dederick)

Wheatland Prairie (Hermitage)

Round Prairie (Humansville)

Britton Prairie (Kahoka SE)

Nine Mile Prairie

(Montgomery City)

Olivers Prairie (Neosho)

Diamond Grove Prairie (Neosho)

Burkhart Prairie (Neosho)

Spurgeon Prairie (Neosho)

Brock Prairie (Neosho)

Joys Prairie (Neosho)

Pools Prairie (Neosho)

White Rock Prairie (Noel)

Burkhart Prairie (Racine)

Lanes Prairie (Redbird)

Pond Prairie (Redbird)

Olivers Prairie (Ritchey)

Swars Prairie (Seneca, Mo.-Okla)

Grand Prairie (Kennet)

Montana :

Tom Lay Prairie (Bozeman)

Belle Prairie (Glendive)

Indian Prairie (Hamilton)

Lone Pine Prairie (Kintla Lakes)

Big Prairie (Kintla Lakes)

Round Prairie (Kintla Lakes)

Lonesome Prairie (Lonesome)

Big Prairie (Silvertip)

Little Indian Prairie

(Thompson Falls)

Big Prairie (Thompson Falls)

Wolf Prairie (Thompson Lakes)
New Mexico:

Cooney Prairie (Alum Mountain)

North Dakota:

North Prairie (Sawyer NE)

New Prairie (Sawyer NE)

Ohio:

Sunbeam Prairie (New Paris)

Oklahoma:

Wildhorse Prairie (Bristow)

Yankee Bill Prairie (Wyandotte)

Sweetwater Prairie (Wyandotte)

Burkhart Prairie (Wyandotte)

Jackson Prairie (Wyandotte)

Swars Prairie (Wyandotte)

Cowskin Prairie (Wyandotte)

Courthouse Prairie (Wyandotte)

Picket Prairie (Kiefer)

Oregon:

Donegan Prairies (sic)

(Abbott Butte)

Abbott Prairie (Abbott Butte)

Long Prairie (Bone Mountain)

Big Prairie (Bone Mountain)

Hall Prairie (Coquille)

Little Summit Prairie (Dayville)

Bull Prairie (Desolation Butte)

Bristow Prairie (Diamond Lake)

Cinder Prairie (Diamond Lake)

Thorn Prairie (Diamond Lake)

Happy Prairie (Diamond Lake)

Bridge Prairie (Diamond Lake)

Dog Prairie (Diamond Lake)

High Prairie (Diamond Lake)

Skookum Prairie

(Diamond Lake)

Beaver Creek Prairie (Dufur)

Smock Prairie (Dufur)

Bottle Prairie (Dufur)

Horse Prairie (Dutchman Butte)

Walker Prairie

(Dutchman Butte) 
Jackass Prairie

(Dutchman Butte)

Walker Prairie (Estacada)

CamasPrairie(Euchre Mountain)

Elos Prairie (Euchre Mountain)

Jeeter Prairie

(Euchre Mountain)

Lost Prairie (Euchre Mountain)

Perkins Prairie (Galice)

Jerome Prairie (Grants Pass)

Threemile Prairie (Heceta Head)

Crane Prairie

(Ironside Mountain)

Tent Prairie (Langlois)

Calf Ranch Prairie (Langlois)

Big Summit Prairie

(Lookout Mountain)

Indian Prairie

(Lookout Mountain)

GrayPrairie (Lookout Mountain)

Lytle Prairie

(Lookout Mountain)

Long Prairie (Maiden Peak)

Indigo Prairie (Marial)

Whitten Prairie (Marial)

Dayton Prairie (McMinnville)

Marks Prairie (Molalla)

Gribble Prairie (Molalla)

Dickey Prairie (Molalla)

Howell Prairie (Mt. Angel)

French Prairie (Mt. Angel)

Elliott Prairie (Mt. Angel)

Gibson Prairie (Mt. Hood)

Crane Prairie (Mt. Hood)

Camas Prairie (Mt. Hood)

Pigeon Prairies (Mt. Jefferson)

Horse Prairie (Post)

Round Prairie (Roseburg)

Round Prairie (Roseburg)

Indian Prairie (Snow Peak)

Fingerboard Prairie

(Three Sisters)

* signifies an unpublished map.
Major Prairie (Waldo Lake)

High Prairie (Waldo Lake)

Crabapple Prairie (Waldo Lake)

Sand Prairie (Waldo Lake)

Joes Prairie (Waldo Lake)

Wilson Prairie (Kimberly)

Brush Prairie (Portland)

Floating Prairie (Richmond)

Long Prairie (Spray)

Gutherie Prairie (Spray)

Pickett Prairie (Tiller)

Rocky Prairie (Cape Ferrelo)

Wild Horse Prairie

(*Collier Butte)

High Prairie (*Collier Butte)

Lately Prairie (*Collier Butte)

Jerome Prairie (*Grants Pass)

Bark Shanty Prairie

(*Port Orford)

Salt Lick Prairie (*Powers)

Calf Ranch Prairie (*Powers)

Flannigan Prairie (*Powers)

Slim Prairie (Diamond Lake)

South Dakota:

Centennial Prairie (Deadwood)

Slate Prairie (Deadwood)

Reynolds Prairie (Deadwood)

Garden Prairie (Ferney)

Gilette Prairie (Harney Peak)

Centennial Prairie (Spearfish)

Centennial Prairie (Sturgis)

Texas:

Peters Prairie (Annona)

Caughorn Prairie (Bassett)

Little Prairie (Bassett)

Hemphill Prairie (Bastrop)

Hills Prairie (Bastrop)

Crafts Prairie (Bastrop)

Saline Prairie (Bullard)

Round Prairie (Fauna)

Blossom Prairie (Hungerford)

Blossom Prairie (Kendleton) 
Tarkington Prairie (Liberty)

Lower Prairie (Liberty)

Dayton Prairie (Liberty)

Batson Prairie (Liberty)

Postoak Prairie (Montague)

Cobb Prairie (Wheelock)

Wheelock Prairie (Wheelock)

Paynes Prairie (Wheelock)

Prairie (*Bessmay)

Batiste Prairie (*Daisetta)

Mayhaw Prairie (*Daisetta)

Batson Prairie (*Daisetta)

Sam's Prairie (*Daisetta)

Stockpen Prairie (*Daisetta)

Blossom Prairie (Kendleton)

Crafts Prairie (Smithville)

Washington:

Fords Prairie (Adna)

Boistfort Prairie (Adna)

Indian Prairie (Airway Heights)

Warner Prairie (Alger)

German Prairie (Alger)

Latourell Prairie (Bridal Veil)

Smith Prairie (Camano)

Bear Prairie (Camas)

Fern Prairie (Camas)

Tyee Prairie (Lake Pleasant)

Caldwell Prairie (Mount Adams)

McEwen Prairie (Potlach)

Mansford Prairie (Stilaguamish)

Lacamas Prairie (Castle Rock)

Cowlitz Prairie (Castle Rock)

Drews Prairie (Castle Rock)

Layton Prairie (Castle Rock)

Grand Prairie (Castle Rock)

Sallal Prairie (Cedar Lake)

Four Mound Prairie (Clayton)

Wild Rose Prairie (Clayton)

Michigan Prairie (Connell)

Smith Prairie (Coupeville)

Ebeys Prairie (Coupeville)

Indian Prairie (Deep Creek)
Wild Rose Prairie (Deer Park)

Half Moon Prairie (Deer Park)

Fivemile Prairie (Deer Park)

Pleasant Prairie (Deer Park)

Peone Prairie (Deer Park)

Chambers Prairie (East Olympia)

Goose Prairie (Elma)

Carstairs Prairie (Elma)

Buck Prairie (Elma)

Horse Prairie (Elma)

Horse Prairie (Elma)

Forks Prairie (Forks)

91 st Division Prairie (FortLewis)

Pleasant Prairie (Greenacres)

South Prairie (Hood River)

Hawks Prairie (Lacey)

Little Chambers Prairie (Lacey)

Fern Prairie (Lackamas Creek)

Smith Prairie (Lake Lawrence)

Rocky Prairie (Maytown)

Bush Prairie (Maytown)

Bush Prairie (Olympia)

Hawks Prairie (Olympia)

Little Chambers Prairie

(Olympia)

Chambers Prairie (Olympia)

Tyee Prairie (Lake Pleasant)

Connells Prairie (Lake Tapps)

Quillayute Prairie (La Push)

Little Prairie (La Push)

Bachelor Prairie (Lincoln)

Ford Prairie (Malone)

Indian Prairie (Medical Lake)

Caldwell Prairie (Mount Adams)

Goose Prairie (Mt. Aix)

Cash Prairie (Mt. Aix)

Swauk Prairie (Mt. Stuart)

Mashel Prairie (Ohop Valley)

Alpha Prairie (Onalaska)

Cowlitz Prairie (Onalaska)

Ahlstroms Prairie (Ozette Lake)

Rooses Prairie (Ozette Lake) 


$\begin{array}{ll}\text { Mannys Prairie (Ozette Lake) } & \text { Bush Prairie (Tenino) } \\ \text { Pe Ell Prairie (Pe Ell) } & \text { Rocky Prairie (Tenino) } \\ \text { Elk Prairie (Pe Ell) } & \text { Violet Prairie (Tenino SW) } \\ \text { Grand Mound Prairie } & \text { Waunch Prairie (Tenino SW) } \\ \quad \text { (Rochester) } & \text { Ruth Prairie (Vail) } \\ \text { Baker Prairie (Rochester) } & \text { Weir Prairie (Weir) } \\ \text { Mima Prairie (Rochester) } & \text { Dalley Prairie (Wickersham) } \\ \text { Jolm Prairie (Shelton) } & \text { Chelatchie Prairie (Yacolt) } \\ \text { Thirteenth Division Prairie } & \text { Yelm Prairie (Yelm) } \\ \quad \text { (Spanaway) } & \text { Ruth Prairie (Yelm) } \\ \text { Fourth Division Prairie } & \text { Weir Prairie (Yelm) } \\ \quad \text { (Spanaway) } & \text { Smith Prairie (Yelm) } \\ \text { Orchard Prairie (Spokane NE) } & \text { Wisconsin: } \\ \text { Pleasant Prairie (Spokane NE) } & \text { Dead Lake Prairie (Arkansaw) } \\ \text { Fivemile Prairie (Spokane NW) } & \text { Prairie du Sac (Blue Mounds) } \\ \text { Moran Prairie (Spokane SE) } & \text { Sand Prairie (Boscobel) } \\ \text { Moran Prairie (Spokane SW) } & \text { Amsterdam Prairie (Galesville) } \\ \text { Moran Prairie (Spokane) } & \text { Decorah Prairie (Galesville) } \\ \text { Fivemile Prairie (Spokane) } & \text { Round Prairie (Ripon) } \\ \text { Orchard Prairie (Spokane) } & \text { Pepin Prairie (Wabasha) } \\ \text { Pleasant Prairie (Spokane) } & \text { Dead Lake Prairie (Arkansaw) } \\ \text { Summit Prairie } & \\ \quad \text { (Steamboat Mountain) } & \text { Wyoming: } \\ \text { Rock Prairie (Tenino) } & \text { Walker Prairie (Dayton) } \\ \text { Frost Prairie (Tenino) } & \text { Canyon Springs Prairie } \\ \text { Viblet (sic) Prairie (Tenino) } & \text { (Sundance) } \\ \text { Mound Prairie (Tenino) } & \end{array}$

False Generics

\begin{abstract}
P
L RAIRIE occurs as a false Generic approximately three times as often as a true generic, yet no more than thirty examples of corresponding pairs of these two classes of terms were found on identical maps, as illustrated by Pine Prairie and Pine Prairie Bay (i. e., bayhead), both on the Ingalls, Arkansas, map, and Sand Prairie (true generic) and Sand Prairie the town (Boscobel, Wisconsin). Nevertheless, in my opinion, most of the false generics discussed below probably were originally connected with correspond-
\end{abstract}


ing true generics. It seems to me that where there is a Prairie View School there should be - or have been - an actual prairie visible from the site of the building; and terms like Prairie Church, High Prairie Church, Prairie Rest Church, Prairie Home, Prairie Home School, and Prairie City seem to strengthen such a conclusion. However, in the vast majority of such instances the evidence is simply lacking.

The names of American schools containing the term prairie, totaling 413 , make up by far the largest single category of false generics. Prairie School itself occurs 35 times; and the same combination preceded by a modifying element, as in Bluff Prairie School, Pleasant Prairie School, Pretty Prairie School, Sandy Prairie School, Lost Prairie School, Bottom Prairie School, Garden Prairie School, Sauk Prairie School, Maine Prairie School, Brush Prairie School, Liberty Prairie School, and Sand Prairie School, totaled 189. There are 188 instances of terms on the model of Prairie College School, such as Prairie View School, Prairie Queen School, Prairie Flower School, Prairie Lawn School, Prairie Center School, Prairiedale School, Prairie Hill School, Prairie Dell School, Prairie Creek School, Prairie Dog School, Prairie Pride School, and Prairie Belle School. Some of the schools have been abandoned, but their names are preserved on the maps: e.g., Prairie School, High Prairie School, Prairie Flower School, Prairie Grove School and Layton Prairie School.

Prairie also occurs 113 times in the names of townships, the second largest category of false generics. Included here are the simple forms Prairie and La Prairie, and terms with preceding modifiers like Vine Prairie, Lick Prairie, Erin Prairie, Boeuf Prairie, Nine Mile Prairie, Sauk Prairie, Lost Prairie, Pine Prairie, and those forms with the prairie elements coming first, such as Prairie Grove, Prairieton, Prairieville, Prairie Creek, Prairie du Chien and Prairie du Long.

Fifty-four towns are named for prairies, either in isolated form or in collocation: for example, Prairie (twice), La Prairie, Prairieton, Prairie View (twice), Prairieville, Prairietown, Prairie City, Prairie Center, Prairie Lea, Prairie Depot, Prairie du Chien, Grand Prairie, Star Prairie, Sand Prairie, East Prairie, West Prairie, Hams Prairie, and Joy Prairie. The names of hamlets and localities like Prairie, String Prairie, and Empire Prairie might be added here. 
The names of 73 churches in the United States make some use of the term prairie. There are three Prairie Churches; twenty-one examples of prairie-plus-name-plus-church, such as Prairie Rest Church, Prairie Creek Church, and Prairie Dell Church; and thirtysix occurrences of the modifier-prairie-church type, like High Prairie Church, Pleasant Prairie Church, Oswego Prairie Church, and Oak Prairie Church.

Prairie combines with the term cemetery twenty-five times. Eighteen of the examples follow the pattern of French Prairie Cemetery, Pink Prairie Cemetery, Pleasant Prairie Cemetery and Mound Prairie Cemetery. There are seven occurrences of the Prairie Home Cemetery type, including Prairie Church Cemetery, Prairie Grove Cemetery, Prairie Lake Cemetery, and Prairieville Cemetery.

Of all the collocations designating natural topographic features, the term which combines most frequently with prairie is creek, the compounds totaling 153. The expression prairie creek is found 98 times. Included in the modifier-prairie-creek type are Lake Prairie Creek, Little Prairie Creek, Muddy Prairie Creek, and the curious Dog Prairie Creek, Horse Prairie Creek and Halfway Prairie Creek. The prairie-name-creek form is illustrated by Prairie Cypress Creek and Prairie du Long Creek.

Among the other topographic false generics are the following, which enter into collocation with prairie itself as the first element (specific examples being given in the reference list at the end of the article): bayou (13 times), bottom, branch (25), brook, fork, hill (9), hollow (14), island, lake (22; see below), mountain (8), outlet, peak, pond, rapids, reef, ridge, river (6), run (6), slough, spring, valley, and the geological term Little Prairie Aa Flow (in the Craters of the Moon National Monument, Idaho). One of the most puzzling of these terms is lake: sometimes it is an actual lake (Chetek, Wis., and Barlow, Ky.); it appears on one map as wetland in the shape of a horseshoe (Henrico, Ark.); and again it seems to be a lake surrounded by wetland (Caroga Lake, New Mexico), or a lake adjoining wetland (Cohasset East, Minn.) - in the last instance it is questionable just how much is prairie and how much is lake. Examples of other prairie combinations with other topographic terms are Pine Prairie Bay (i. e., bayhead), Two Prairie Bayou, Hill Prairie Church, Belle Prairie Cut-Off, Prairie Dell Dam, Prairie Flower Draw, Harrell Prairie Hill, Prairie Run Hollow, 
Prairie Lake Hollow, Mount Prairie Hollow, Flat Prairie Island, Vine Prairie Lake, Indian Prairie Lake, Lost Prairie Lake, Eightmile Prairie Mountain, First Prairie Mountain, Second Prairie Mountain, Mountain Prairie Church, Prairie Dell Pond, Prairie Rapids Church, Little Prairie Ridge, Muddy Prairie Run, Maine Prairie Slough, Round Prairie Spring, Prairie Valley School and Prairie Valley Church.

The following cultural terms form compounds with prairie, each expression functioning as the second element: -(s)burg, camp, center, chapel (6), city, depot, ditch (10), farm, home, pipe line, road, -ton, top, and -ville. Other compounds of the same general group are Indian Prairie Canal, Prairie Creek Ditch, Shaker Prairie Ditch, Cowlitz Prairie Grange, South Prairie Lookout, Prairie Center Mission, Burnt Prairie Post Office, Eden Prairie Post Office, Prairie View Post Office, Crane Prairie Reservoir, Smith Prairie Road, and Maine Prairie (Site) (sic.). There seem to be only two counties in the United States which are named Prairie, one in Arkansas and the other in Montana.

Direct French influence is illustrated by the forms Prairie du Long (township), Prairie du Rocher (town, and township), Prairie du Ronde School, Prairie du Ronde (township), La Prairie (occurring twice as a town), and the well-known Prairie du Chien.

The following terms are more complicated in form than the preceding illustrations of false generics: Connels Prairie Battle Monument; Lower Brice Prairie School; Crane Prairie Guard Station, and Camas Prairie Ranger Station (there being six similar stations); Pine Prairie Oil Field; Prairie Mountain Spring Branch; Prairie Lake Heights School; Prairie Farm Heights School; East, and West, Prairie Grove Schools; Swars Prairie Church of Christ; Swars Prairie Baptist Church; West Fire Prairie Creek; North Fork Prairie Dog Creek; Prairie Creek Redwoods State Park; Green Prairie Fish Lake; Rail Prairie Town Hall; and Twin-Crane Prairie Trail.

According to the map evidence discussed above, French influence on the American vocabulary was heaviest in the Mississippi Valley area, from which it apparently branched principally to the Pacific Northwest, the eastern part of the North Central states and also to the southeastern Gulf region. As might be expected, the twenty- 
four states which have true prairie generics also have false generics. Among this particular group Illinois has more false generics than any other state, 212 in number, and thereby justifies its nickname as the "Prairie State" even if it cannot do so on the basis of the number of its true generics. Missouri has the second largest number of false generics, 173. Of the nine other states in which only false generics are found, Colorado has 5, Nebraska 21, Iowa 26, Michigan 16, Tennessee 5, Alabama 4; and perhaps Kentucky, Pennsylvania, and New York - having only one false generic each - could more accurately be called the fringe states. At any rate, the term prairie is used in designating places and topographic features in the greater portion of the country. Therefore, when the early French explorers originated prairie generics, they made a significant contribution to the American language.

The false generics referred to and the topographical maps on which they occur are as follows: (* indicates an unpublished map.)

False Generics ws. True Generics

Pine Prairie Bay and Pine

Prairie (Ingalls, Ark.)

Sand Prairie

(Boscobel, Wisconsin)

Prairie View School

Rapid City, 1 SE, S. D.)

Prairie Church (Marion, Ill.)

High Prairie Church

(Niangua, Mo.)

Prairie Rest Church

(Pelahatchie, Miss.)

Prairie Home (Dalton City, Ill.)

Prairie Home School

(Kearney, Mo.)

Prairie City (Papinsville, Mo.)

Schools

Prairie School (Gillett, Ark.)

Bluff Prairie School (Weott,Cal.)

Pleasant Prairie School

(Fort Totten, N. Dak.)

Pretty Prairie School

(Mechanicsburg, Ohio)
Sandy Prairie School (Gay Hill, Texas)

Lost Prairie School

(Palestine, Texas)

Bottom Prairie School (Mt. Sterling, Ill.)

Garden Prairie School

(Fulton, Mo.)

Sauk Prairie School

(Norma, N. Dak.)

Maine Prairie School

(Dozier, Cal.)

Brush Prairie School

(West Frankfort, Ill.)

Liberty Prairie School

(Edwardsville, Ill.)

Sand Prairie School

(Rockford, Ill.)

Prairie College School

(Pana, Ill. (5))

Prairie View School

(Hamburg, Wis.)

Prairie Queen School

(Red Lake Falls, Minn.) 
Prairie Flower School (Silex,Mo.)

Prairie Lawn School (Delan, Ill.)

Prairie Center School (Clinton,Ill

Prairie Center School

(Clinton, Ill.)

Prairiedale School, (Elgin, Ill.)

*Prairie Hill School

(Nashville, Ill.)

Prairie Dell School

Cissna Park, Ill.)

Prairie Creek School

(Mackinaw, Ill.)

Prairie Dog School

(McDonald, Kans.)

Prairie Pride School

(Ferney, S. Dak.)

Prairie Belle School

(Pukwana, S. Dak.)

(Abandoned:)

Prairie School (Utica, Mo.)

High Prairie School

California, North, Mo.)

Prairie Flower School

(Winterset, Iowa)

Prairie Grove School

(California South, Mo.)

Layton Prairie School

Castle Rock, Wash.)

\section{Townships}

Prairie (Booneville, Ark.)

LaPrairie (Brentford, S.Dak.(3))

Vine Prairie

(Winslow, Ark.-Okla.)

Lick Prairie,

(Mt. Carmel, Ill.-Ind.)

Erin Prairie (Deer Park, Wis.)

Boeuf Prairie

(Ft. Necessity, La.)

Nine Mile Prairie (Fulton, Mo.)

Sauk Prairie (Tolley, N. Dak.)

Lost Prairie (Fulton, Ark.)

Pine Prairie (Turkey Creek, La.)
Prairie Grove

(Winslow, Ark.-Okla.)

*Prairieton (Pimento, Ind.)

Prairieville (Nebo, Mo.)

*Prairie Creek (Pimento, Ind.)

Prairie du Chien (Waukon, Wis.)

Prairie du Long, (Baldwin, Ill.)

\section{Towns:}

Prairie (Ash Fork, Arizona)

Prairie (Wickersham, Wash.)

La Prairie (Camp Point, Ill.)

*Prairieton (Pimento, Ind.)

Prairie View

(Magazine, Mt., Ark. (2))

Prairieville (Hastings, Mich.)

Prairietown (Edwardsville, Ill.)

Prairie City (Avon, Ill. (2))

Prairie Center (Hazen, Ark. (2))

Prairie Lea (San Marcos, Tex.)

Prairie Depot (Elmore, Ohio)

Prairie du Chien

(Prairie du Chien, Wis.)

Grand Prairie (Dallas, Tex.)

Star Prairie

(New Richmond, Wis.)

Sand Prairie (Boscobel, Wis.)

East Prairie (Charleston, Mo.)

West Prairie (Ferryville, Wis.)

Hams Prairie (Fulton, Mo.)

Joy Prairie (Arenzville, Ill.)

Prairie (Baldwin, Ill.)

String Prairie (Flatonia, Tex.)

Empire Prairie (Stanberry, Mo.)

\section{Churches:}

Prairie Church (Marion, Ill.)

Prairie Rest Church

(Pelahatchie, Miss.)

*Prairie Creek Church

(Pimento, Ind.)

Prairie Dell Church

(Watseka, Ill. (2)) 
High Prairie Church (Niangua, Mo.)

Pleasant Prairie Church (Rush City, Wis.) Oswego Prairie Church

(Aurora South, Ill.)

Oak Prairie Church (Hazen, Ark.)

\section{Cemeteries:}

French Prairie Cemetery (Ione, Ark.)

Pink Prairie Cemetery (Erie.,Ill.)

Pleasant Prairie Cemetery (Bates City, Mo.)

Mound Prairie Cemetery (Hudson, Minn.)

Prairie Home Cemetery (Topeka, Kans.)

Prairie Church Cemetery (Kahoka, Mo.)

Prairie Grove Cemetery (Utica, N. Y.)

Prairie Lake Cemetery Chetek, Wis.)

Prairieville Cemetery (Sterling, Ill.)

\section{Creeks:}

Prairie Creek (Caddo, Ala.)

Lake Prairie Creek (Blue Lake, Cal.)

Little Prairie Creek (Slagle, La.) Muddy Prairie Creek (Lancaster, Ohio)

Dog Prairie Creek (Diamond Lake, Oregon)

Horse Prairie Creek Cape Ferrelo, Oregon)

Halfway Prairie Creek (Cross Plains, Wis.) Prairie Cypress Creek (Indian Bay, Ark.)
Prairie du Long Creek (Waterloo, Ill.)

Other Topographic Terms:

Prairie Bayou (Hazen, Ark.) Prairie Bottom (Cherokee, Ala.) Prairie Branch (Alma, Ark.(15))

Prairie Brook (Bessemer Iron District, Ala.) Prairie Fork (San Antonio, Cal.) Prairie Hill (Euchre Mt., Oregon (2))

Prairie Hollow (Topaz, Mo.)

Prairie Island (Felsenthal, Ark.-La. (4))

Prairie Lake (Rich Hill, Mo. (intermittent))

Prairie Mountain (Ute Peak, Colo. (4))

Prairie Outlet (Greenville,Ohio)

Prairie Peak (Olmstead,Ark.(2))

Prairie Ponds (Holley, Fla.)

Prairie Rapids

Prairie Reef (Silvertip, Mont.)

Prairie Ridge

Prairie River Cohasset East, Minn. (5)

Prairie Run (Oil City, Pa. (5)

Prairie Slough

Prairie Spring Carthage N. E., New Mex.)

Prairie Valley (Waco, Tex.)

Little Prairie Aa Flow (Craters of the Moon National Monument)

Prairie Lake (Chetek, Wis.)

Prairie Lake (Barlow, Ky.)

Prairie Lake (Henrico, Ark.)

Prairie Lake Caroga Lake, New Mex.)

Prairie Lake (Cohasset East, Minn.) 
Twomile Prairie Lake

Inverness N. E., Fla.)

Weekiwachee Prairie Lake

(Port Richey, Fla.)

Squirrel Prairie Lake

(Masaryktown, Fla.)

*Pine Prairie Bay (bayhead)

(Ingalls, Ark.)

Hill Prairie Church

(Bastrop, Tex.)

Belle Prairie Cut-Off

(Mileston, Miss.)

Prairie Dell Dam

(Tomahawk, Wis.)

Prairie Flower Draw

(Crows Landing, Cal.)

Harrell PrairieHill (Forest,Miss.)

PrairieRun Hollow (Monett,Mo.)

Prairie Lake Hollow

(Long Lane, Mo.)

Mount Prairie Hollow

(Montier, Mo.)

Flat Prairie Island (Grand Lake

East N. W., La.)

Vine Prairie Lake

(Mulberry, Ark. (5))

Indian Prairie Lake

(Snow Peak, Oregon)

Lost Prairie Lake

Palestine, Tex.)

Eightmile Prairie Mountain

(Port Orford, Oregon)

First Prairie Mountain

(Port Orford, Oregon)

Second Prairie Mountain

(Port Orford, Oregon)

Mountain Prairie Church

(Mt. Sterling, Ill.)

Prairie Dell Pond

(Tomahawk, Wis.)

Prairie Rapids Church

(McCord, Wis.)
Little Prairie Ridge

Pecan Island, La.)

Muddy Prairie Run

(Lancaster, Ohio)

Maine Prairie Slough

(Dozier, Cal.)

Round Prairie Spring

(Saddle Butte, Wash.)

Prairie Valley School

(Hermitage, Miss.)

Prairie Valley Church

(Gallatin, Mo.)

Cultural Terms:

Prairiesburg (Farley, Iowa)

Prairie Camp (Lowell, Oregon)

Prairie Center

(Coupeville, Wash.)

Prairie Chapel (Kansas, Ill.)

Prairie City (Papinsville, Mo.)

Prairie Depot (Elmore, Ohio)

Prairie Ditch (Delphos, Ohio)

Prairie Farm

(St. Charles, Mich. (5))

Prairie Home (Dalton City, Ill.)

Prairie Pipe Line (Buckner, Mo.)

Prairie Road (Wheeling, Ill.)

Prairieton (Pimento, Ind.)

Prairie Top

(Salinas Peak, New Mex.)

Prairieville

(Denham Springs, La.)

Indian Prairie Canal (Basinger

S. W. and N. W., Fla.)

Prairie Creek Ditch (Puxico,Mo.)

Shaker Prairie Ditch

(Oaktown, Ind.-Ill.)

Cowlitz Prairie Grange

(Castle Rock, Wash.)

South Prairie Lookout

(Cedar Lake, Wash.) 
Prairie Center Mission (Blessing, Tex.)

Burnt Prairie Post Office (Engield, Ill.)

Eden Prairie Post Office (Minneapolis, Minn.)

Prairie View Post Office (Hempstead, Tex.)

Crane Prairie Reservoir (Maiden Peak, Oregon)

Smith Prairie Road (Lake Lawrence, Wash.)

Maine Prairie (Site), (sic) (Dozier, Cal.)

Direct French Influence:

Prairie du Long(NewAthens, Ill.)

Prairie du Rocher (Renault, Ill.-Mo.)

Prairie du Ronde School (Opelousas, La. (2))

Prairie du Ronde (Schoolcraft, Mich.)

La Prairie (Shopiere,Wis. (2))

Prairie du Chien (Prairie du Chien, Wis.)

Complicated Terms:

Connels Prairie Battle Monument, (Lake Tapps, Wash.)

Lower Brice Prairie School (La Crescent, Va.)
Crane Prairie Guard Station (Maiden Peak, Oreg.)

Camas Prairie Ranger Station, (Mt. Jefferson, Oregon)

Pine Prairie Oil Field (Turkey Creek, La.)

Prairie Mountain Spring Branch (Llano, Tex.)

Prairie Lake Heights School (Barron, Wis.)

Prairie Farm Heights School (Downing, Wis.)

East, and West, Prairie Grove Schools (Sweet Springs, Mo.)

Swars Prairie Church of Christ (Seneca, Mo.-Okla.)

Swars Prairie Baptist Church (Seneca, Mo.-Okla.)

West Fire Prairie Creek (Blue Springs, Mo.)

North Fork Prairie Dog Creek (Achilles, Kans.)

Prairie Creek Redwoods State Park (Orick, Cal.)

Green Prairie Fish Lake (Belle Prairie, Minn.)

Rail Prairie Town Hall (Belle Prairie, Minn.) Twin-Crane Prairie Trail (Maiden Peak, Oregon) 